\title{
MINI-REVIEW
}

\section{Proton Pump Inhibitors and Helicobacter Pylori-Associated Pathogenesis}

\author{
Tadashi Hagiwara, Ken-ichi Mukaisho*, Takahisa Nakayama, Takanori Hattori, \\ Hiroyuki Sugihara
}

\begin{abstract}
The fact that long-term use of proton pump inhibitors (PPIs) aggravates corpus atrophic gastritis in patients with Helicobacter pylori infection has been proven clinically and experimentally. Corpus atrophic gastritis is a known risk factor for gastric cancer. Therefore, gastric neoplasia might be associated with the long-term use of PPIs. One of the causes of worsening corpus atrophic gastritis, leading to the development of adenocarcinoma, might be bacterial overgrowth under conditions of hypochlorhydria. The production of potentially carcinogenic $\mathrm{N}$-nitrosocompounds by nitrosating organisms under conditions of hypochlorhydria might be associated with carcinogenesis. Interactions between bile acids, $\mathrm{pH}$, and $\boldsymbol{H}$. pylori might also contribute to carcinogenicity, especially in patients with gastro-esophageal reflux disease (GERD). The concentration of soluble bile acids, which have bactericidal or chemorepellent properties toward $H$. pylori, in gastric contents is considerably higher in patients undergoing continuous PPI therapy than in healthy individuals with normal acid production. Under these circumstances, H.pylori might colonize the stomach body rather than the pyloric antrum. Hypergastrinemia induced by PPI administration might promote the development of gastric cancer. Because the main cause of corpus atrophic gastritis is $H$.pylori infection, and not PPI administration, $H$.pylori infection should be eradicated before starting long-term PPI therapy.
\end{abstract}

Keywords: Helicobacter pylori - proton pump inhibitors - gastric cancer - corpus atrophic gastritis - GERD - gatrin

Asian Pac J Cancer Prev, 16 (4), 1315-1319

\section{Introduction}

Helicobacter pylori $(H$. pylori) is a microbial species that specifically colonizes the gastric epithelium (Polk et al., 2010). H. pylori infections are probably acquired in childhood and adolescence (Kuipers et al., 1993). Infected individuals develop coexisting gastritis, which typically persists for decades, coupling $H$. pylori and its human host into a dynamic and prolonged equilibrium (Polk et al., 2010). In a study conducted by Graham et al., healthy volunteers deliberately infected with a well-characterized laboratory strain of $H$. pylori developed inflammation of the acute infectious phase in both the proximal and distal stomach mucosa (Graham et al., 2004). When colonization becomes persistent, infection results in chronic gastritis lasting for many years, possibly life-long. H.pylori is the strongest known risk factor for gastric cancer. It has been estimated that $H$. pylori colonization increases the risk of gastric cancer by approximately 10 -fold, and H.pylori has been designated a class I carcinogen by the World Health Organization (IARC Monographs on the Evaluation of Carcinogen Risk to Humans, 1994). Intestinal-type adenocarcinoma, the more prevalent form of gastric cancer, is believed to develop via a series of histological steps. The cancer progresses from chronic gastritis to atrophic gastritis, intestinal metaplasia, and dysplasia (Correa et al., 1975). Uemura et al. demonstrated that $H$. pylori-infected patients who have corpus-predominant atrophic gastritis with intestinal metaplasia have a high risk of developing adenocarcinoma (Uemura et al., 2001).

The use of proton pump inhibitors (PPIs) to treat peptic ulcers and gastro-esophageal reflux disease (GERD) has increased substantially over the past two decades. Furthermore, widespread use of non-steroidal anti-inflammatory drugs in populations at a risk for gastro-duodenal side effects is another common indication for PPI therapy (Torgownik et al., 2008; Hawkey et al., 1998). It has been widely accepted that long-term use of PPIs aggravates corpus atrophic gastritis in patients with H. pylori infections (Kuipers et al., 1995; Kuipers et al., 1996; Garcia at al., 2006). Although the exact mechanism underlying this effect has not been determined, longterm PPI therapy in $H$. pylori-infected patients has been suggested to increase the risk for gastric cancer. Thus, a potential association might exist between gastric neoplasia and long-term use of PPIs.

In this review, we discuss the mechanism underlying corpus-predominant gastritis, which leads to gastric cancer 
related to PPI therapy in $H$. pylori-positive patients, including in those with GERD.

\section{Relationship between acid-suppressive therapy and corpus atrophic gastritis}

In the 1970s, corpus atrophic gastritis was observed in duodenal ulcer patients undergoing vagotomy (Meikle et al., 1976). Although H. pylori had not yet been identified as the causative organism, most of these patients were probably infected with $H$. pylori, as evidenced by the presence of refractory duodenal ulcers. Subsequently, aggravation of corpus atrophic gastritis was reported in many $H$. pylori-infected GERD patients undergoing long-term PPI therapy. Kuipers et al. evaluated GERD patients from two separate cohorts; one group was treated with omeprazole (OPZ) and the other group, with a fundoplication. The incidence of corpus atrophic gastritis was very low in both patient groups in the absence of $H$. pylori infection. However, a significantly higher number of $H$. pylori-infected patients undergoing OPZ therapy developed corpus atrophic gastritis than H.pylori-infected patients treated with a fundoplication, over an average period of 5 years (Kuipers et al., 1996). Long-term use of lansoprazole, as well as OPZ, aggravates corpus atrophic gastritis in $H$. pylori-infected patients (Geboes et al., 2001).

Suitable animal models to study the histological changes induced by $\boldsymbol{H}$. pylori

Animal models may be used to understand the pathogenesis of human diseases. Several murine models for H.pylori infection have been reported in the literature. Initially, long-term colonization of the murine stomach by $H$. pylori was difficult to achieve. However, in the 1990s, some researchers reported that $H$. felis and $H$. pylori strain SS1 could colonize the mouse stomach and cause lymphocytic gastritis but not peptic ulcers or gastric cancer (Lee et al., 1990; Lee et al., 1997). In 1991, Yokota et al. (1991) demonstrated that $H$. pylori could colonize the stomach of Mongolian gerbils. Subsequently, some researchers reported that the gastric pathology of $H$. pylori-infected Mongolian gerbils was similar to that of human patients exhibiting peptic ulcers, atrophic gastritis, intestinal metaplasia (Matsumoto et al., 1997; Honda et al., 1998a), and gastric adenocarcinoma (Honda et al., 1998b; Wang et al., 1998; Watanabe et al., 1998; Franco et al., 2005). Therefore, Mongolian gerbils are excellent model organisms to study $H$. pylori infection, because the gross and histological findings mimic the lesions induced by $H$. pylori infection in the human gastric mucosa.

\section{Omeprazole (OPZ) administration promotes corpus atrophic gastritis in Mongolian gerbils infected with $H$. pylori: a study by our group}

In a previous study, we successfully demonstrated that long-term PPI administration promotes corpus atrophic gastritis in Mongolian gerbils with $H$. pylori infection
(Hagiwara et al., 2011). Briefly, we divided the gerbils into four groups: H.pylori (ATCC43504) was inoculated into the OPZ (omeprazole) $+\mathrm{Hp}$ (H.pylori) and Hp groups, a PPI (OPZ) was administered to the OPZ+Hp and OPZ groups and the control group received no treatment. At the end of the 6-month experiment, corpus atrophic gastritis and adenocarcinomas were significantly more common in the OPZ+Hp group than in the other groups. However, one of the important findings of this study was that long-term OPZ administration in the absence of $H$. pylori infection did not induce corpus atrophic gastritis. In another experiment using male rats, neither corpus atrophic gastritis nor neoplastic lesions developed after administration of OPZ over a period of 1 year (Hagiwara et al., 2007). These findings suggested that $H$. pylori infection, and not long-term PPI administration, was the direct cause of corpus atrophic gastritis leading to the development of adenocarcinoma (Fox et al., 2011; Hagiwara et al., 2011; 2012).

\section{Intragastric $\mathrm{pH}$ and the gastric microbiota}

Conventional wisdom espoused the dogma that the stomach was a sterile organ. This was attributed to the acidic conditions $(\mathrm{pH}<4)$ prevalent in the stomach (Hill et al., 1985). However, over the last 30 years, with the discovery of H.pylori (Marshall et al., 1984; Warren et al., 1983), it has been established that the stomach supports a bacterial community with hundreds of phylotypes (approximate species-level taxa) (Bik et al., 2006; Andersson et al., 2008; Li et al., 2009). Drug-induced inhibition or reduction of gastric acid secretion due to parietal cell loss caused by H. pylori infection can lead to hypochlorhydria or even achlorhydria, thereby increasing the risk of bacterial overgrowth and possible detrimental infections throughout the gastrointestinal tract (Martinsen et al., 2005; Cover TL et al., 2009). Bacterial overgrowth under conditions of hypochlorhydria $(\mathrm{pH}>4)$ might be responsible for the worsening corpus atrophic gastritis observed in patients with $H$. pylori infection undergoing long-term treatment with PPIs.

\section{Possible mechanism of gastric carcinogenesis under conditions of hypochlorhydria}

$H$. pylori infection and the associated changes in the stomach alter the ecological niche inhabited by the gastric microbiota. However, the gastric microbiota also competes with $H$. pylori for a gastric niche, and therefore, it might play an important role in the progression of the disease (Sheh et al., 2013). Before the discovery of $H$. pylori infection, Correa proposed that gastric carcinogenesis is the result of a chain of events triggered by the onset of hypochlorhydria and the colonization of the stomach by nitrosating bacteria (Correa, 1984; Correa, 1992). Nitrosating organisms can produce potentially carcinogenic $\mathrm{N}$-nitrosocompounds by combining nitrite and other organic nitrogen compounds present in the gastric juice. These $\mathrm{N}$-nitrosocompounds are known to cause malignancies of the digestive tract (Xu et al., 1993; 
Ziebarth et al., 1997).

Transgenic insulin-gastrin (INS-GAS) mice, which exhibit hypergastrinemia, develop atrophic gastritis, hypochlorhydria, and gastrointestinal intraepithelial neoplasia upon infection with $H$. pylori (Wang et al., 2000). Gastric atrophy and hypochlorhydria lead to non-helicobacter microbiota overgrowth. Lofgren et al. reported that $H$. pylori monoassociation induced cancer in INS-GAS mice, but the absence of gastric colonization with enteric microbes significantly inhibited the development of gastric cancer in the INS-GAS mice (Lofgren et al., 2011). These findings suggest that microbiota overgrowth might increase the risk of gastric cancer in $H$. pylori-infected patients with gastric atrophy and hypochlorhydria.

\section{Interactions between bile acids, $\mathbf{p H}$, and $H$. pylori in GERD patients}

The use of PPIs for the treatment of GERD has increased substantially over the past two decades. We recently proposed that interactions between bile acids, $\mathrm{pH}$, and $H$. pylori might be responsible for exacerbated corpus atrophic gastritis in $H$. pylori-positive patients with GERD undergoing long-term treatment with PPIs. Duodeno-gastric reflux, which is composed of bile, occurs even in healthy individuals, and bile reflux is increased in patients with GERD (Dixon et al., 2001). Diluted human plasma and bile acids have been found to function as potent chemoattractants and chemorepellents, respectively, toward $H$. pylori (Worku et al., 2004). Although only taurine conjugates, with a pKa of 1.8-1.9, are soluble in an acidic environment, glycine conjugates, with a pKa of 4.3-5.2, as well as taurine-conjugated bile acids, are soluble in the presence of PPI (Kuipers et al., 1995; Nair at al., 1971; Stamp et al., 2002). Thus, the concentration of soluble bile acids in the gastric contents of patients with GERD after continuous PPI therapy is considerably higher than that in healthy individuals with normal acid production. In the distal stomach, the soluble bile acids, at high concentrations, function as a bactericide or chemorepellent toward H. pylori. In contrast, the mucous layer in the proximal stomach has an optimal bile concentration that forms chemotactic gradients with the plasma components that are required to direct $H$. pylori to the epithelial surface. H. pylori may then colonize the stomach body rather than the pyloric antrum, which may explain the occurrence of corpus-predominant pan gastritis after PPI therapy in $H$. pylori-positive patients with GERD (Mukaisho et al., 2014).

\section{Effects of hypergastrinemia on gastric cancer development}

Infection with $H$. pylori increases gastrin expression (Shafaghi et al., 2013; Sordal et al., 2013), and acid suppression therapy leads to hypergastrinemia in nearly all patients receiving long-term PPI therapy (Orlando et al., 2007). Gastrin has a trophic effect on the gastrointestinal tract and acts as a growth and differentiation factor
(Tsukamoto et al., 2011). However, it is still unclear whether hypergastrinemia promotes the development of gastric adenocarcinoma. In patients with hypergastrinemia, such as those with Zollinger-Ellison syndrome or pernicious anemia, enterochromaffin-like (ECL) cell hyperplasia occasionally develops but rarely leads to carcinoids. In animal models, INS-GAS transgenic mice, which exhibit hypergastrinemia, with $\mathrm{H}$. felis or H.pylori (strain SS1) infection develop gastric cancer (Wang et al., 2000; Fox et al., 2003), but this not accompanied by ECL cell hyperplasia (Wang et al., 2000). Hypergastrinemia in rats results in the development of ECL cell carcinoids (Havu et al., 1986; Havu et al., 1990; Mattsson et al., 1991). As mentioned previously, our study demonstrated that the serum gastrin levels were higher in the OPZ+Hp group than in the other groups and that the OPZ+Hp group developed gastric adenocarcinoma (Hagiwara et al., 2011). These findings suggest that hypergastrinemia might promote the development of gastric cancer in patients infected with $H$. pylori undergoing long-term treatment with PPIs. Watson et al. hypothesized that gastrin might not lead to cancer development on its own in humans, but it might function as an important cofactor once premalignant changes are triggered (Watson et al., 2006).

\section{Conclusion}

In this review, we discussed the possible mechanism underlying the development of atrophic corpus gastritis leading to adenocarcinoma in patients with $H$. pylori infection undergoing long-term PPI therapy. Although many studies initially raised doubts about the safety of long-term PPI administration, several studies have now shown that the main cause of worsening corpus atrophic gastritis leading to the development of adenocarcinoma is not long-term PPI administration, but $H$. pylori infection. Therefore, it is recommended that patients being considered for long-term PPI therapy should be tested for $H$. pylori infection. If infection is detected, the pathogen must be eradicated before commencement of treatment.

\section{References}

Andersson AF, Lindberg M, Jakobsson H, et al (2008). Comparative analysis of human gut microbiota by barcoded pyrosequencing. PLoS One, 3, 2836.

Bik EM, Eckburg PB, Gill SR, et al (2006). Molecular analysis of the bacterial microbiota in the human stomach. Proc Natl Acad Sci USA, 103, 732-7.

Correa P, Haenszel W, Cuello C, et al (1975). Archer M. A model for gastric cancer epidemiology. Lancet, 2, 58-9.

Correa P (1984). Chronic gastritis as a cancer precursor. Scand $J$ Gastroenterol Suppl, 104, 131-6.

Correa P (1992). Human gastric carcinogenesis: a multistep and multifactorial process-- First American Cancer Society Award Lecture on Cancer Epidemiology and Prevention. Cancer Res, 52, 6735-40.

Cover TL, Blaser MJ (2009). Helicobacter pylori in health and disease. Gastroenterology, 136, 1863-73.

Dixon MF, Neville PM, Mapstone NP, et al (2001). Bile reflux gastritis and Barrett's oesophagus: further evidence of a role for duodenogastro-oesophageal reflux? Gut, 49, 359-63.

Franco AT, Israel DA, Washington MK, et al (2005). Activation 
of beta-catenin by carcinogenic Helicobacter pylori. Proc. Natl Acad Sci USA, 102, 10646-51.

Fox JG, Rogers AB, Ihrig M, et al (2003). Helicobacter pyloriassociated gastric cancer in INS-GAS mice is gender specific. Cancer Res, 63, 942-50.

Fox JG, Kuipers EJ (2011). Long-term proton pump inhibitor administration, H pylori and gastric cancer: lessons from the gerbil. Gut, 60, 567-8.

García Rodríguez LA, Lagergren J, Lindblad M, et al (2006). Gastric acid suppression and risk of oesophageal and gastric adenocarcinoma: a nested case control study in the UK. Gut, $\mathbf{5 5}, 1538-44$.

Geboes K, Dekker W, Mulder CJ, et al (2001). Long-term lansoprazole treatment for gastro-oesophageal reflux disease: clinical efficacy and influence on gastric mucosa. Aliment Pharmacol Ther, 15, 1819-26.

Graham DY, Opekun AR, Osato MS, et al (2004). Challenge model for Helicobacter pylori infection in human volunteers. Gut, 53, 1235-43.

Hagiwara T, Mukaisho M, Ling ZQ, et al (2007). Rebamipide contributes to reducing adverse effects of long-term administration of omeprazole in rats. Dig Dis Sci, 52, 988-94.

Hagiwara T, Mukaisho M, Nakayama T, et al (2011). Long-term proton pump inhibitor administration worsens atrophic corpus gastritis and promotes adenocarcinoma development in Mongolian gerbils infected with Helicobacter pylori. Gut, 60, 624-30.

Hagiwara T, Mukaisho M,Nakayama T, et al (2012). Experimental strategies for induction of gastric adenocarcinomas under long-term proton pump inhibitor administration and Helicobacter pylori infection. Asian Pac J Cancer Prev, 13, 3005-6

Havu N (1986). Enterochromaffin-like cell carcinoids of gastric mucosa in rats after life-long inhibition of gastric secretion. Digestion, 35, 42-55.

Havu N, Mattsson H, Ekman L, et al (1990). Enterochromaffinlike cell carcinoids in the rat gastric mucosa following longterm administration of ranitidine. Digestion, 45, 189-95.

Hawkey CJ, Karrasch JA, Szczepanski L, et al (1998). Omeprazole compared with misoprostol for ulcers associated with nonsteroidal antiinflammatory drugs. Omeprazole versus Misoprostol for NSAID-induced Ulcer Management (OMNIUM) Study Group. N Engl J Med, 338, 727-34.

Hill M (1985). Normal and pathological microbial flora of the upper gastrointestinal tract. Scand J Gastroenterol Suppl, 111, 1-6.

Honda S, Fujioka T, Tokieda M, et al (1998a). Gastric ulcer, atrophic gastritis, and intestinal metaplasia caused by Helicobacter pylori infection in Mongolian gerbils. Scand $J$ Gastroenterol, 33, 454-60.

Honda S, Fujioka T, Tokieda M, et al (1998b). Development of Helicobacter pylori-induced gastric carcinoma in Mongolian gerbils. Cancer Res, 58, 4255-9.

IARC Monographs on the Evaluation of Carcinogen risk to humans: some industrial chemicals, (1994) No. 60, Lyon: International Agency for Research on Cancer.

Kuipers EJ, Pena AS, Kamp GV, et al (1993). Seroconversion for Helicobacter pylori. Lancet, 342, 328-31.

Kuipers EJ, Uyterlinde AM, Pena AS, et al (1995). Increase of Helicobacter pylori-associated corpus gastritis during acid suppressive therapy: implications for longterm safety. Am J Gastroenterol, 90, 1401-6.

Kuipers EJ, Lundell L, Klinkenberg-Knol EC, et al (1996). Atrophic gastritis and Helicobacter pylori infection in patients with reflux esophagitis treated with omeprazole or fundoplication. $N$ Engl J Med, 334, 1018-22.

Lee A, Fox AJ, Otto G, et al (1990). A small animal model of human Helicobacter pylori active chronic gastritis. Gastroenterology, 99, 1315-23.

Lee A, O'Rourke J, De Ungria MC, et al (1997). A standardized mouse model of Helicobacter pylori infection: introducing the Sydney strain. Gastroenterology, 112, 1386-97.

Li XX, Wong GL, To KF, et al (2009). Bacterial microbiota profiling in gastritis without Helicobacter pylori infection or non-steroidal anti-inflammatory drug use. PLoS One, 4, 7985 .

Lofgren JL, Whary MT, Ge Z, et al (2011). Lack of commensal flora in Helicobacter pylori-infected INS-GAS mice reduces gastritis and delays intraepithelial neoplasia. Gastroenterology, 140, 210-20.

Marshall BJ, Warren JR (1984). Unidentified curved bacilli in the stomach of patients with gastritis and peptic ulceration. Lancet, 1, 1311-15.

Martinsen TC, Bergh K, Waldum HL (2005). Gastric juice: a barrier against infectious diseases. Basic Clin Pharmacol Toxicol, 96. 94-102.

Matsumoto S, Washizuka Y, Matsumoto Y, et al (1997). Induction of ulceration and severe gastritis in Mongolian gerbil by Helicobacter pylori infection. J Med Microbiol, 46, 391-7.

Mattsson H, Havu N, Brautigam J, et al (1991). Partial gastric corpectomy results in hypergastrinemia and development of gastric enterochromaffinlike-cell carcinoids in the rat. Gastroenterol, 100, 311-9.

Meikle DD, Taylor KB, Truelove SC, et al (1976). Gastritis duodenitis, and circulating levels of gastrin in duodenal ulcer patients before and after vagotomy. Gut, 17, 719-28.

Mukaisho K, Hagiwara T, Nakayama T, et al (2014). Potential mechanism of corpus-predominant gastritis after PPI therapy in Helicobacter pylori -positive patients with GERD. World J Gastroenterol, 20, 11962-5.

Nair PP, Kritchenvski D (1971). The Bile Acids: Chemistry, Physiology and Metabolism. Chemistry. New York: Plenum Press, 1.

Orlando LA, Lenard L, Orlando RC, et al (2007). Chronic hypergastrinemia: causes and consequences. Dig Dis Sci, 52, 2482-9.

Polk DB, Peek Jr RM (2010). Helicobacter pylori: gastric cancer and beyond. Nat Rev Cancer, 10, 403-14.

Shafaghi A, Mansour-Ghanaei F, Joukar F, et al (2013). Serum gastrin and the pepsinogen I/II ratio as markers for diagnosis of premalignant gastric lesions. Asian Pac J Cancer Prev, 14, 3931-6.

Stamp DH (2002). Three hypotheses linking bile to carcinogenesis in the gastrointestinal tract: certain bile salts have properties that may be used to complement chemotherapy. Med Hypotheses, 59, 398-405.

Sheh A, Fox JG (2013). The role of the gastrointestinal microbiome in Helicobacter pylori pathogenesis. Gut Microbes, 4, 505-31.

Sordal O, Waldum H, Nordrum IS, et al (2013). The gastrin receptor antagonist netazepide (YF476) prevents oxyntic mucosal inflammation induced by Helicobacter pylori infection in Mongolian gerbils. Helicobacter, 18, 397-405.

Targownik LE, Metge CJ, Leung S, et al (2008). The relative efficacies of gastroprotective strategies in chronic users of nonsteroidal anti-inflammatory drugs. Gastroenterology. 134, 937-44.

Tsukamoto H, Mizoshita T, Sasaki M, et al (2011). Longterm high-dose proton pump inhibitor administration to Helicobacter pylori-infected Mongolian gerbils enhances neuroendocrine tumor development in the glandular stomach. Asian Pac J Cancer Prev, 12, 1049-54.

Uemura N, Okamoto S, Yamamoto S, et al (2001). Helicobacter pylori infection and the development of gastric cancer. $N$. 
Engl J Med. 345, 784-9.

Wang TC, Fox JG (1998). Helicobacter pylori and gastric cancer: Koch's postulates fulfilled? Gastroenterol, 115, 780-3.

Wang TC, Dangler CA, Chen D, et al (2000). Synergistic interaction between hypergastrinemia and Helicobacter infection in a mouse model of gastric cancer. Gastroenterology, 118, 36-47.

Warren JR, Marshall BJ (1983). Unidentified curved bacilli on gastric epithelium in active chronic gastritis. Lancet, 1, 1273-5.

Watanabe T, Tada M, Nagai H, et al (1998). S. Helicobacter pylori infection induces gastric cancer in Mongolian gerbils. Gastroenterol, 115, 642-8.

Watson SA, Grabowska AM,El-Zaatari M, et al (2006). Gastrin -active participant or bystander in gastric carcinogenesis? Nature Rev Cancer, 6, 936-46.

Worku ML, Karim QN, Spencer J, et al (2004). Chemotactic response of Helicobacter pylori to human plasma and bile. J Med Microbiol, 53, 807-11.

Xu GP, Reed PI (1993). N-nitroso compounds in fresh gastric juice and their relation to intragastric $\mathrm{pH}$ and nitrite employing an improved analytical method. Carcinogenesis, 14, 2547-51.

Yokota K, Kurebayashi Y, Takayama Y, et al (1991). Colonization of Helicobacter pylori in the gastric mucosa of Mongolian gerbils. Microbiol Immunol, 35, 475-80.

Ziebarth D, Spiegelhalder B, Bartsch H (1997). N-nitrosation of medicinal drugs catalysed by bacteria from human saliva and gastro-intestinal tract, including Helicobacter pylori. Carcinogenesis, 18, 383-9. 